\title{
Design and validation of biologically inspired spiculated breast lesion models utilizing structural tissue distortion
}

Premkumar Elangovan, Elena Mihalas, Majdi Alnowami, Kenneth C. Young, David R. Dance, et al.

Premkumar Elangovan, Elena Mihalas, Majdi Alnowami, Kenneth C. Young, David R. Dance, Victoria Cooke, Louise Wilkinson, Rosalind M. GivenWilson, Matthew G. Wallis, Kevin Wells, "Design and validation of biologically inspired spiculated breast lesion models utilizing structural tissue distortion," Proc. SPIE 10573, Medical Imaging 2018: Physics of Medical Imaging, 105730B (9 March 2018); doi: 10.1117/12.2293421

SPIE. Event: SPIE Medical Imaging, 2018, Houston, Texas, United States 


\title{
Design and validation of biologically-inspired spiculated breast lesion models utilizing structural tissue distortion.
}

\author{
Premkumar Elangovan ${ }^{\mathrm{a}, \mathrm{b}}$, Elena Mihalas ${ }^{\mathrm{a}}$, Majdi Alnowami ${ }^{\mathrm{a}, \mathrm{h}}$, Kenneth C. Young ${ }^{\mathrm{b}, \mathrm{c}}$, David \\ R. Dance ${ }^{\mathrm{b}, \mathrm{c}}$, Victoria Cooke ${ }^{\mathrm{d}}$, Louise Wilkinson ${ }^{\mathrm{i}}$, Rosalind M. Given-Wilson ${ }^{\mathrm{e}}$, Matthew G. \\ Wallis $^{\mathrm{f}}$, Kevin Wells ${ }^{\mathrm{a}}$
}

${ }^{\mathrm{a} C e n t r e ~ f o r ~ V i s i o n, ~ S p e e c h ~ a n d ~ S i g n a l ~ P r o c e s s i n g, ~ U n i v e r s i t y ~ o f ~ S u r r e y, ~ G u i l d f o r d, ~ G U 2 ~}$

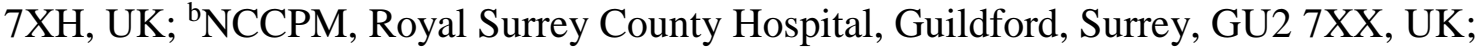
'Department of Physics, University of Surrey, Guildford, GU2 7XH, UK; ${ }^{\mathrm{d} J a r v i s ~ B r e a s t ~}$ Screening and Diagnostic Centre, Guildford GU1 1LJ, UK; ${ }^{\mathrm{e} D e p a r t m e n t ~ o f ~ R a d i o l o g y, ~ S t . ~}$ George's Healthcare NHS Trust, Tooting, London SW17 0QT, UK; 'Cambridge Breast Unit, Cambridge University Hospitals NHS Foundation Trust, Cambridge CB2 0QQ, UK and

${ }^{9}$ NIHR Cambridge Biomedical Research Centre, Cambridge CB2 0QQ, UK; ${ }^{\text {hN }}$ uclear

Engineering Department, King Abdulaziz University, Jeddah, KSA; ${ }^{\mathrm{O} O x f o r d ~ B r e a s t ~ I m a g i n g ~}$ Centre, Oxford University Hospitals NHS Trust, OUH NHS UK

\begin{abstract}
The use of conventional clinical trials to optimise technology and techniques in breast cancer screening carries with it issues of dose, high cost and delay. This has motivated the development of Virtual Clinical Trials (VCTs) as an alternative in-silico assessment paradigm. However, such an approach requires a set of modelling tools that can realistically represent the key biological and technical components within the imaging chain. The OPTIMAM image simulation toolbox provides a complete validated end-to-end solution for VCTs, wherein commonly-found regular and irregular lesions can be successfully and realistically simulated. As spiculated lesions are the second most common form of solid mass we report on our latest developments to produce realistic spiculated lesion models, with particular application in Alternative Forced Choice trials. We make use of sets of spicules drawn using manually annotated landmarks and interpolated by a fitted 3D spline for each spicule. Once combined with a solid core, these are inserted into 2D and tomosynthesis image segments and blended using a combination of elongation, rotational alignment with background, spicule twisting and core radial contraction effects. A mixture of real and simulated images (86 2D and 86 DBT images) with spiculated lesions were presented to an experienced radiologist in an observer study. The latest observer study results demonstrated that $88.4 \%$ of simulated images of lesions in 2D and $67.4 \%$ of simulated lesions in DBT were rated as definitely or probably real on a six-point scale. This presents a significant improvement on our previous work which did not employ any background blending algorithms to simulate spiculated lesions in clinical images.
\end{abstract}

Keywords: Phantom, lesion, model, simulation, mammography.

\section{INTRODUCTION}

Modern screening for breast cancer has undergone major technological development with the move away from film-screen to digital and tomosynthesis technologies. However, this has not been accompanied by a similar pace of change in the approaches used for the assessment of new screening technologies and methodologies. The traditional clinical trial, which involve 1000s of participants represents a bottleneck to delivering new technologies and techniques into clinical practice. This has motivated the development of new in-silico methods, known as virtual clinical trials $(\mathrm{VCTs})^{1}$.

Medical Imaging 2018: Physics of Medical Imaging, edited by Joseph Y. Lo

Taly Gilat Schmidt, Guang-Hong Chen, Proc. of SPIE Vol. 10573, 105730B

(C) 2018 SPIE · CCC code: $1605-7422 / 18 / \$ 18 \cdot$ doi: $10.1117 / 12.2293421$ 
The OPTIMAM image simulation toolbox ${ }^{2}$ is a complete end-to-end VCT pipeline that can generate synthetic masses $^{3,4}$ or calcifications ${ }^{5}$ and insert these into unprocessed clinical digital mammography images or hybrid models of breast tissue ${ }^{6}$. These are then processed using an image acquisition tool that can currently simulate either the Hologic Selenia ${ }^{2}$ or the Siemens MAMMOMAT Inspiration ${ }^{7}$ imaging systems in 2D and tomosynthesis operating modes. The images are then post-processed using vendor-specific image processing/reconstruction software.

Spiculated lesions are the second most common form of solid mass seen in screening, and should therefore be represented within a VCT framework. Prior work on simulating spiculated masses ${ }^{8}$ has focussed on using a mathematical approach which can be complex and time consuming, and can have limited levels of visual realism. One factor that may affect realism is the complex manner in which spiculated masses interact with the local tissue architecture. The only major work in this area to examine this effect ${ }^{9}$ modelled tissue distortion using a wavelet based approach. This elegant approach produced promising results, although some types of background and mass could not be adequately represented.

The purpose of this latest work is to develop methods of generating spiculated mass models demonstrating stateof-the-art levels of realism. This has been achieved using biologically-inspired spicule morphology, and modelling the appearance of commonly seen local structural distortions to enhance integration with local tissue architecture.

\section{METHODS AND MATERIALS}

\subsection{Generating biologically-inspired spiculated masses}

Spiculated mass models were generated by examining clinical cases present in digital breast tomosynthesis (DBT) images. By scrolling through the DBT image stack, the centre of the spicule as seen on each slice was manually annotated to form a set of 3D landmarks, and then 3D splines were fitted to the landmarks to define a spicule 'skeleton' (Figure 1a-c). The width of the spicule observed in the DBT stack was also measured at several key points. A 3D horn structure could then be fitted using an empirically observed power law ${ }^{10}$ to model the change in width with distance from the lesion core Figure 1d. Then random distortions were applied to the cones to reduce the geometric appearance. Early experiments demonstrated that assigning spicules with the same density as glandular tissue produced objects that were unrealistically bright. Therefore, the internal structure of the spicule horn manifolds were filled with hair-like strands with each hair given the density of glandular tissue, so that spicule density could be controlled by varying the packing fraction of the resulting strands. Collections of spicules were then attached to solid lesion cores created using diffusion limited aggregation (DLA) ${ }^{3,4}$ as shown in Figure 1e-f.

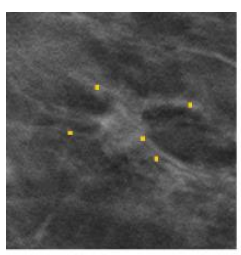

(a)

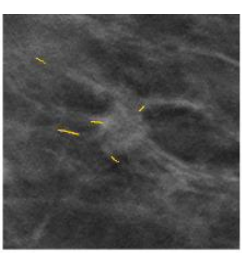

(b)

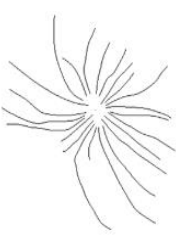

(c)

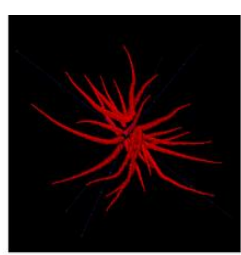

(d)

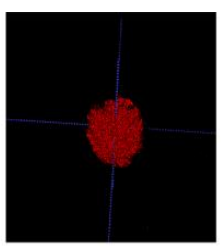

(e)

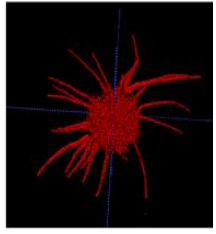

(f)

Fig. 1. Schematic representation of the creation of the spiculated mass 


\subsection{Modelling structural distortion and appearance blending}

A series of experiments was undertaken using our synthetic breast phantoms ${ }^{6}$ to understand the ways in which tissues can appear to distort in the local vicinity of a mass, and so attempt replication of these observed distortions and twists. The approach taken was primarily aimed at producing a realistic appearance as seen in clinical images, and so may not necessarily be representative of actual distortions present in real breast tissue. Empirical observation suggests that lesions tend to have a subtle distortion that aligns with the main axis or orientation of the underlying tissue texture. There are then further local twisting effects where the spicules interact with local tissue texture. Following experimentation and comparison with patterns seen in 2D and DBT clinical images, it was found that the approach described below provided a visually realistic method for integrating the spiculated mass with its local environment using insertion methods ${ }^{4}$.

Using a set of in-house modelling tools, distortions were introduced that represent four key steps to achieve realistic blending of the appearance of the spiculated mass with the local background tissue. These include (i) elongation, or stretching of the lesion, (ii) alignment of the lesion model with the local tissue architecture, (iii) spicule twisting and (iv) core contraction effects, as illustrated in Figure 2.

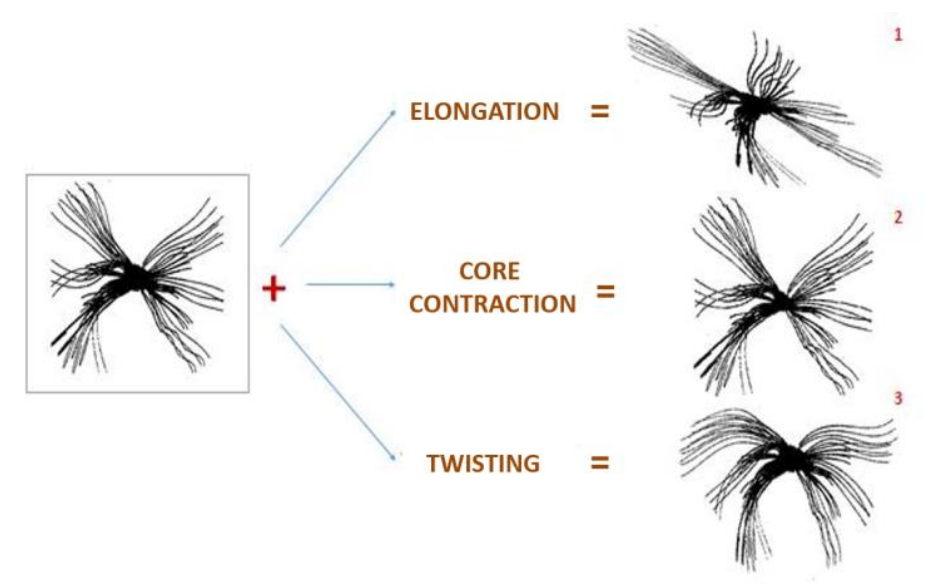

Fig. 2. Demonstration of the deformation provided by the elongation, core contraction and twisting effects when applied to the binary mask of a particular lesion.

\subsection{Elongation and alignment}

A unidirectional elongation was applied to the spiculated lesion model in order to distort the pseudo-elipsoidal shape of the lesion's core. The magnitude of the effect was carefully controlled to preserve a visually realistic level of distortion between the lesion and its spicules. First, the simulated spiculated lesion was projected onto a 2D plane from which a binary mask was extracted. The lesion center was then computed as the centroid of a resulting 2D lesion template. The major orientation of the mass was calculated by applying Principal Component Analysis to the set of points forming this template. This provided the data covariance matrix, which was decomposed into an eigenvalue and an eigenvector matrix. Thus, the principal eigenvector associated with the highest eigenvalue determined the major orientation. 


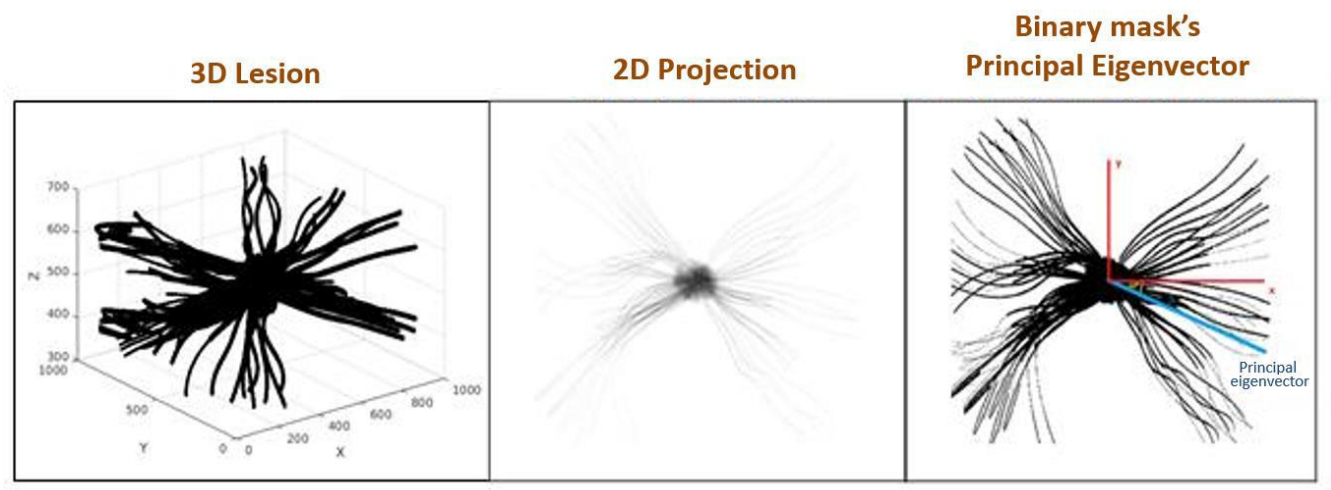

Fig. 3. The main steps of elongation simulation: 1) the spiculated lesion in 3D (left); 2) its projection on a 2D plane (middle); 3) the lesion binary mask orientation (right).

Once the main orientation was known, the 3D lesion was rotated, so that the principal eigenvector became aligned with the insertion volume's $x$-axis (red horizontal line depicted in the right most image of Figure 3), and afterwards it is was elongated in the $x$ direction by an arbitrary amount. The projection of two levels of distorted lesions are displayed in Figure 4. Note that a further rotation is then applied in order to align the spiculated lesion model with the orientation of the background radiological texture. Visual inspection of the 2D projected tissue texture at the insertion location was used to define the underlying texture direction to facilitate this alignment. Mass insertion into clinical images was then performed using template multiplication ${ }^{2}$. The $3 \mathrm{D}$ lesion model was then resized and transformed in a cube of size [1000x1000x1000]. Thus, when the lesion is projected onto a 2D image plane, it occupied a square region of size typically ranging from 3.5 to $5.0 \mathrm{~cm}$. Note that in practice very fine spicule ends will not be visible once the lesion is inserted so that in effect the apparent lesion model is significantly smaller than the actual model size.

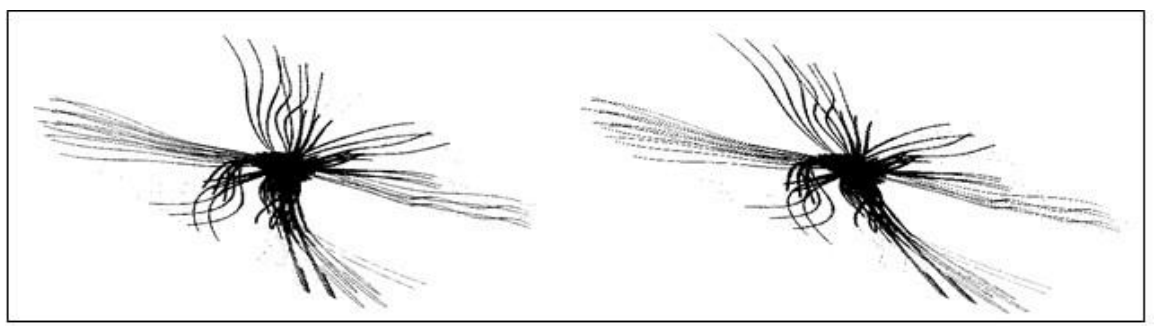

Fig. 4. Two projections of variable elongation with the right-hand model elongated twice as much as the left-hand lesion model

\subsection{Core contraction}

The core contraction effect is a non-linear distortion, which depends on the distance of each point from the lesion's center. In other words, points shift towards the lesion center in relation to their original distance from the lesion's center. For this purpose, it was convenient to shift the image origin from the top left corner to the center and to transform the Cartesian coordinates into polar coordinates.

In order to generate a normalised mask that can be used to implement the core contraction effect across a variety of lesion models, the maximum radius $R$ within the insertion region, which is the distance between the center of the lesion (coincident with image center) and the image corner, was calculated. At this point, it was possible to build two core contraction masks, namely the normalized radius mask Rnorm and the normalized arctangent mask for contraction (equations 1,2). 
$\operatorname{Rnorm}_{i, j}=\frac{\text { radius }_{i, j}}{R}$

Contraction $_{i, j}=\frac{\operatorname{atan}\left(\text { Rnorm }_{i, j}\right)}{\max \left\{\operatorname{atan}\left(\text { Rnorm }_{i, j}\right)\right\}}$

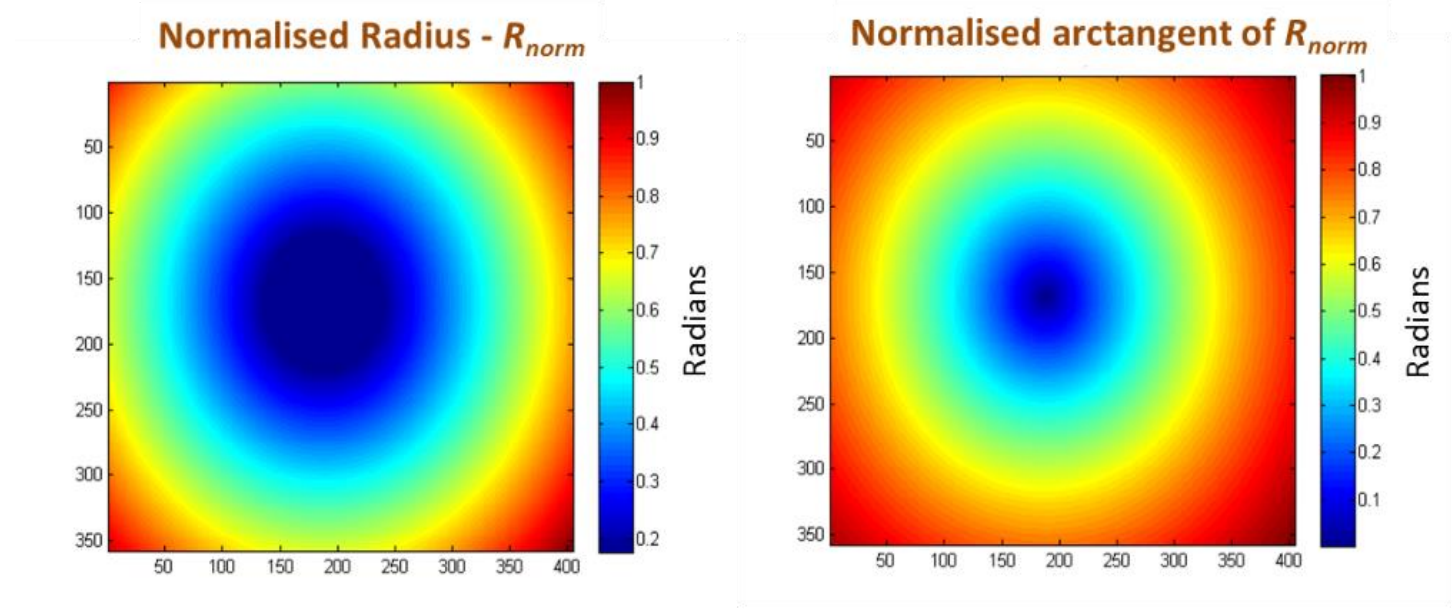

Fig. 5. (Left) The mask containing the normalized radii of image points and (right) the mask described by the normalized arctangent of the normalized radii

The main objective, when simulating the core contraction effect, was to obtain a 2D matrix (mask) with null values close to the image borders and progressively increasing values while approaching the image center. In fact, if the null transformation assumption is not guaranteed along the image borders, obvious discontinuities will be introduced at the insertion location boundaries. Thus, it was necessary to create a mask similar to the normalized arctangent mask but with reversed radial values. To achieve this the normalized arctangent mask values were transformed by applying equation 3 .

$$
\text { Mask }_{i, j}=\operatorname{var} * \frac{\text { Contraction }_{i, j}}{\text { Rnorm }_{i, j}}\left(1-\text { Contraction }_{i, j}\right)
$$

Equation 3 facilitated the creation of a core contraction mask (see Figure 6a, whose values had a steep trend from null values towards the highest value determined by the scalar var (see Figure 6b). However, this also produces a pronounced sucking effect in the central region, which causes shrinkage of lesion's core (see Figure 6c). Thus, it was necessary to preserve the information in the center of the insertion region by delineating a protection zone where the values cannot fall below a default threshold (see Figure 6d-f).

The core contraction mask thus obtained was combined with the original radius matrix element-by-element and the reconverted coordinates interpolated in order to create the final transformation (see Figure $6 \mathrm{c}, \mathrm{f}$ ).

An alternative approach to blending the lesion with its surroundings involved inserting the original lesion into a DBT stack of images ${ }^{2}$ and apply the core contraction simultaneously to the insertion volume such that the blending between the two models was increased (see Figure 7). This created a realistic but slightly different visual blending appearance with the background. 


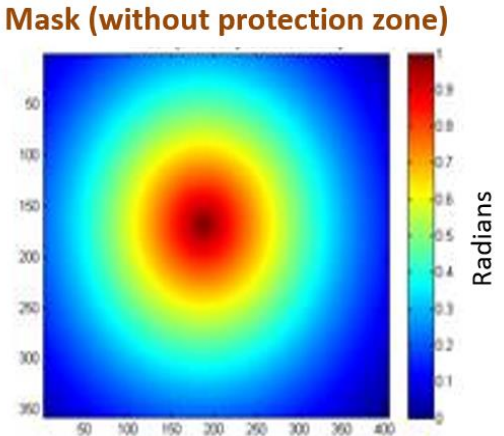

(a)

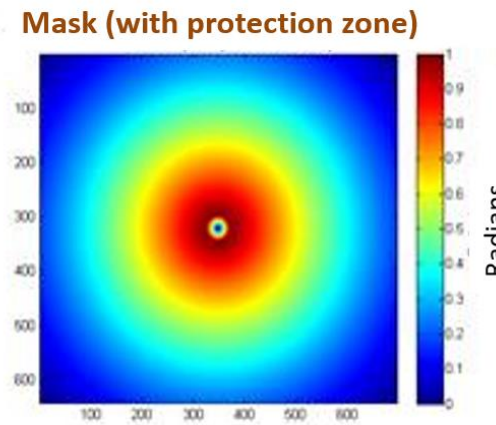

(d)

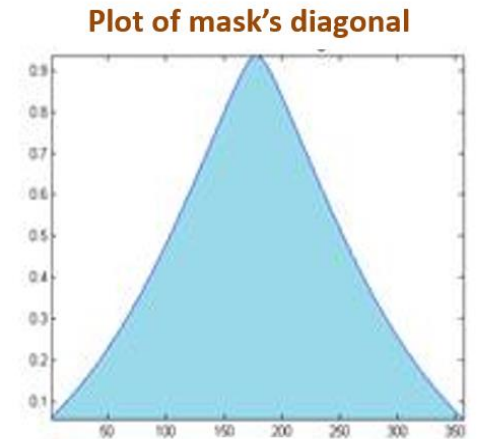

(b)

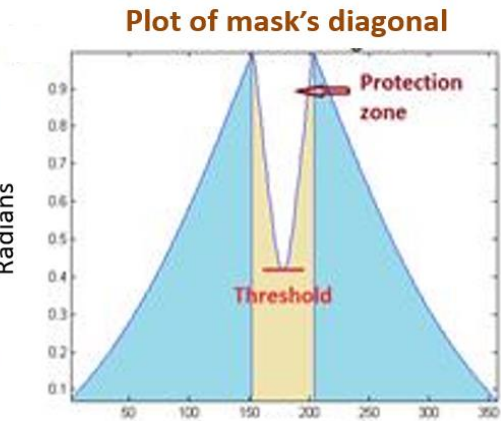

(e)

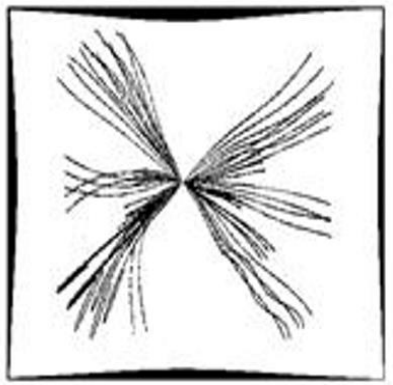

(c)

2D Projection of lesion

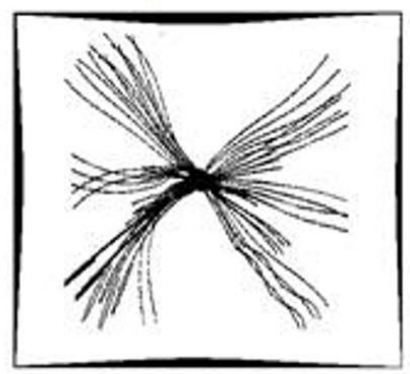

(f)

Fig. 6. The core contraction masks (a) and (d), the diagonal profile of the contraction masks (b) and (e) and the binary masks of the lesion after the contraction effect has been applied (c) and (f). Images are shown for both cases of image center protection (top row - without protection zone; bottom row - with protection zone)
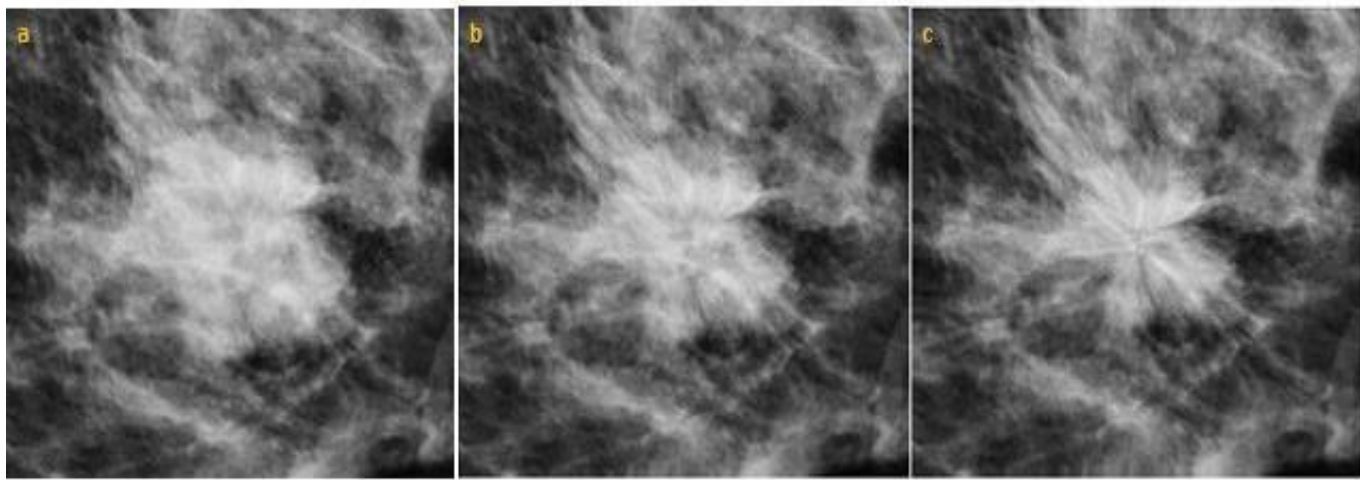

Fig. 7. a) Illustration of the effect of applying core contraction represented as a pulling force towards the center of a DLA mass embedded in a real clinical background image. (a) original mass inserted into breast background tissue (b) effect of core contraction applied to mass seen in (a); (c) doubling the effective contraction seen in (b) such that 'hairy' or spicule-like structures appear at the mass margin. 


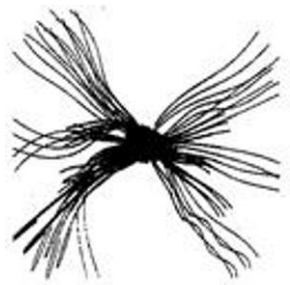

(a)

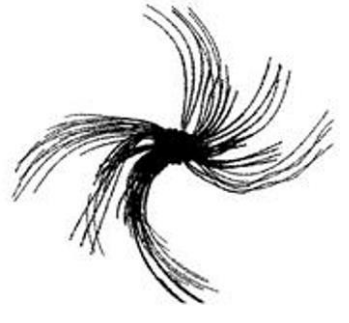

(b)

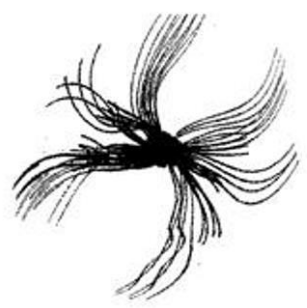

(d)
Symmentric twisting

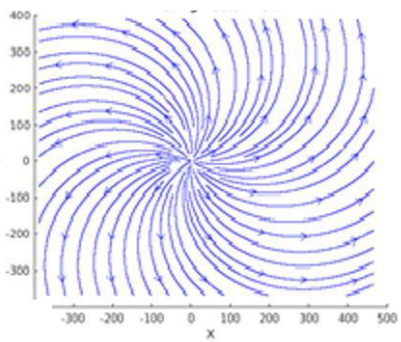

(c)

Asymmentric twisting

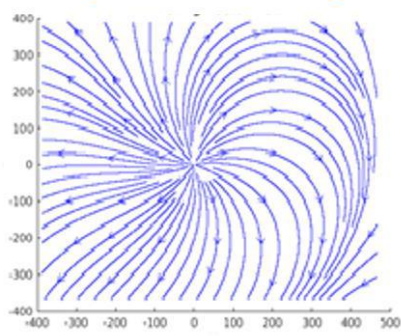

(e)

Fig. 8. Distorted images and the vector fields obtained when applied to a binary lesion model (a) symmetric twisting (b)-(c) and asymmetric twisting (d)-(e). Note that the vector field was built using the polar coordinates of points. Therefore, the figure displays the displacement of points laying originally on rectilinear rays radiating from the lesion center.

\subsection{Twisting}

The twisting effect is a complex transformation, which manipulates a $2 \mathrm{D}$ grid of points in order to obtain a symmetric or asymmetric angular shifting with respect to the lesion. However, a symmetric distortion is rarely observed in screen-detected spiculated lesions, whereas a more random twisting of the tissue surrounding the lesion is more likely to be seen. Thus, it was necessary to develop a twisting algorithm that applied a variable radial twisting effect without discontinuities. This is illustrated in Figure 8. In order to obtain generate this effect, a smooth variation of values inside the twist field was applied using cubic spline interpolation. First it was necessary to define the maximum angular distortion permitted by defining a range of allowed angular displacements (example [- $\pi / 2: \pi / 2]$ ). Then, initially, the image was divided into columns with each column assigned an angular shift extracted from the previously established range of values (see Figure 9a). At this point, the extracted shift values are interpolated along all image points (see Figure 9d-f). However, this produced significant discontinuities visible at the column borders (see Figure 9c) if the selected points are kept constant within each column (see Figure 9b). This was addressed by using a smooth shift function as shown in Figure 9d$\mathrm{f}$ to achieve a smooth non-linear variable twisting action. 


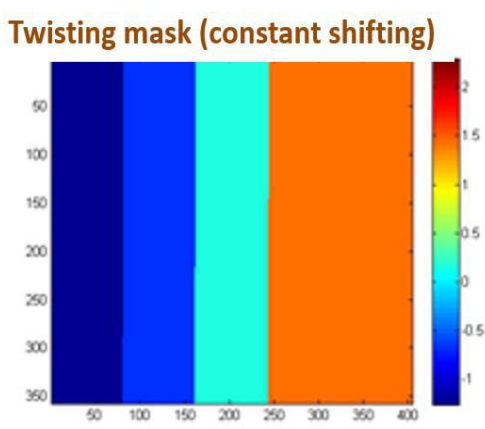

(a)

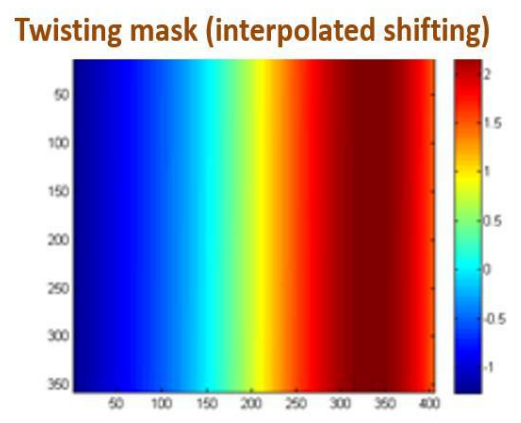

(d)

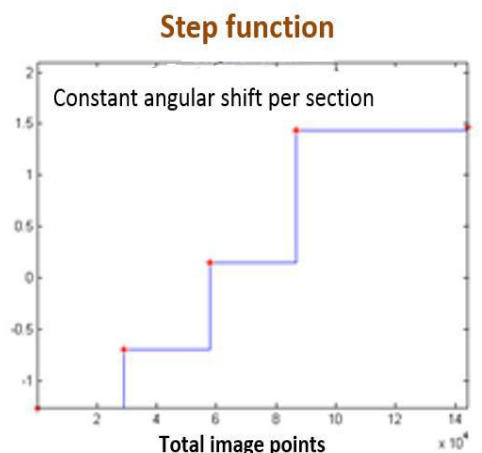

(b)

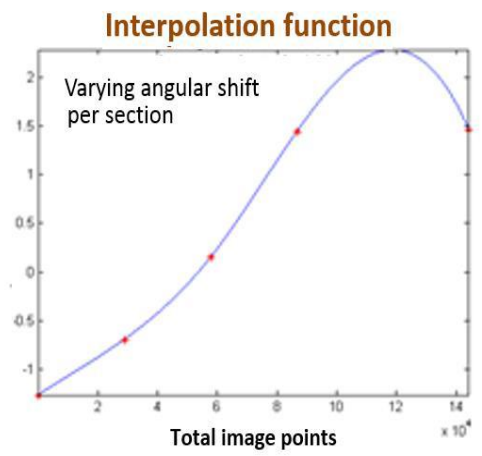

(e)

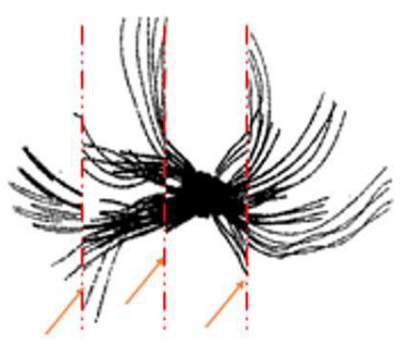

(c)

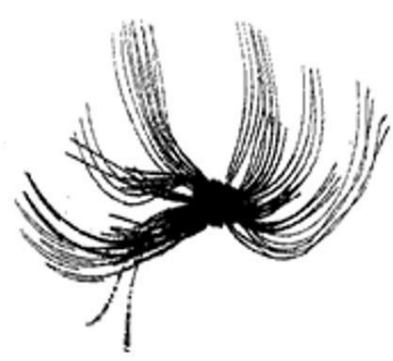

(f)

Fig. 9. The difference between a constant (a)-(c) and an interpolated (d)-(e) angular shift assignment. The twisting masks (twist matrix) (a) and (d) display the values inside the four vertical sections of the image, whereas the functions (b) and (e) represent the same values aligned in a vector of size [M*N]. The lesion's binary masks (c) and (f) encompass the applied distortions.

\subsection{Observer Study}

A pre-pilot study was first conducted using a small set of images followed by an extended observer study with a larger image dataset. A Consultant Radiologist with 12 years screening experience participated in both studies. For the pre-pilot study, eleven lesion models were inserted into a mixture of 2D and DBT image data acquired in the Hologic system (Hologic Inc, Massachusetts, USA). The simulated spiculated lesions were inserted into real clinical images using template multiplication ${ }^{3,4}$. The observer was then asked to rate these on a 3-point scale as either 'real', 'equivocal', or 'simulated'. The pilot study results demonstrate $100 \%$ of $2 \mathrm{D}$ and $64 \%$ of DBT images were rated as 'definitely or probably real'. The size of these initial simulated spiculated masses ranged between $7-9 \mathrm{~mm}$ and the breast region shown to the observer was limited to $3 \times 3 \mathrm{~cm}^{2}$. This is because the spiculated lesions were predominantly modelled for use in $4 \mathrm{AFC}$ type observer studies using image patches of this size. All images were presented in ideal viewing conditions (<6LUX) using a high-resolution monitor (Barco 5MP).

For the extended observer study, a total of 43 spiculated lesions were simulated and inserted into real clinical images again using template multiplication ${ }^{3,4}$. The feedback from the pilot study was used to produce more realistic looking spiculated lesions. This included removing the presence of non-realistic radial core distortions, ensuring presence of a clear DBT plane displaying central lesion shape and ensuring sufficient background tissue distortion at the lesion's core location. The simulated lesions were inserted in the same location in both $2 \mathrm{D}$ and DBT projection images of each clinical case. The 2D images were processed using the $2 \mathrm{D}$ image processing software and the DBT projections were reconstructed using a reconstruction algorithm employing filtered back projection technique using Hologic post processing tools. Subsequently, 43 real screen-detected spiculated 2D and DBT images were selected from the OPTIMAM Image Database ${ }^{11}$ for the study. The real images with 
spiculated lesions were chosen to reflect the range of spiculated lesions produced by the simulation model for the study. The real and simulated images were randomly interleaved resulting in a total of 86 2D and $86 \mathrm{DBT}$ images. The images were shown to the same experienced radiologist who had viewed the pre-pilot study, who was asked to assess the images by rating the appearance of the lesions, but now using a 6 point rating scale, where ' 1 ' being 'Extremely confident that the mass is real' and ' 6 ' being 'Extremely confident that the mass is simulated'.

\section{RESULTS AND DISCUSSION}

The images used in the extended study were generated using several lesion models. Inserting these into a variety of different clinical tissue backgrounds using the aforementioned blending process with template multiplication ${ }^{3,4}$ has produced visually realistic results as shown in Figure 10. Figure 7 also shows a standard DLA mass subject to the distortion process and inserted into a clinical image.

The results of the extended observer study are shown in Figures 11-12. It can be seen that $88.4 \%$ of simulated 2D images and $67.4 \%$ of simulated DBT images were rated as realistic by the radiologist. Similarly, $88.4 \%$ of real 2D images and $97.6 \%$ of real DBT images were rated as realistic by the radiologist. The number of images rated equally good in both 2D and DBT were $60.4 \%$ for simulated and $86 \%$ for real images. This is a significant improvement on the previous observer study results ${ }^{10}$ conducted with simulated lesions without adding blending effects as shown in Table 1.
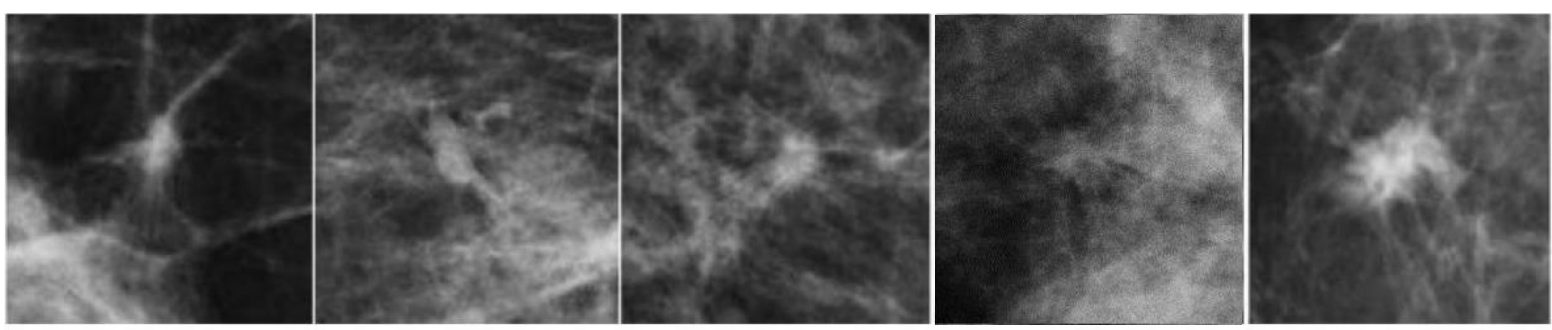

Fig. 10. Examples of simulated lesions inserted with distortion into clinical images
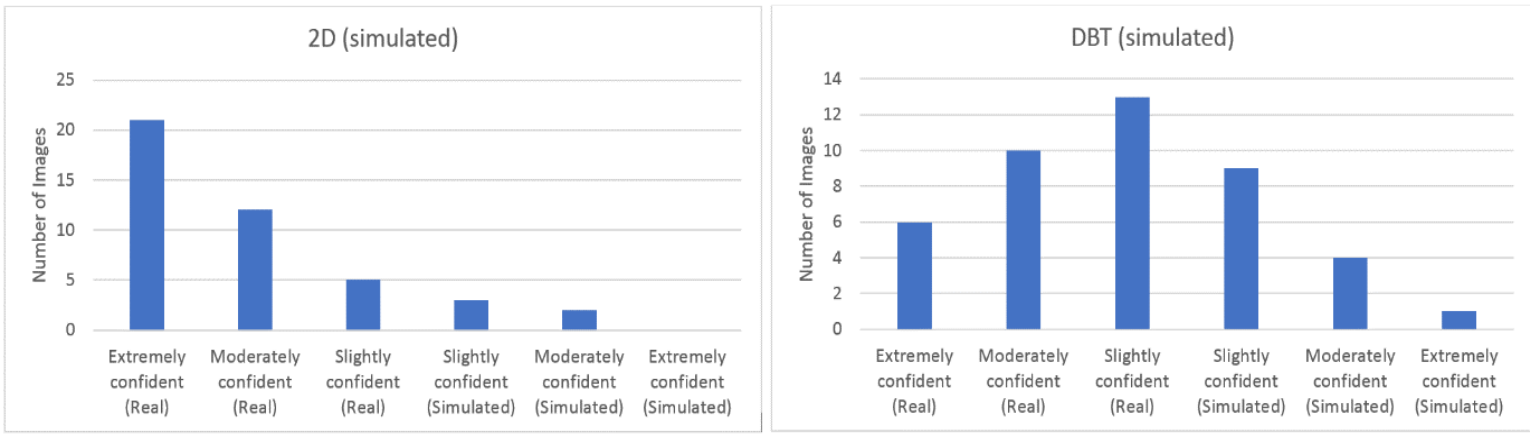

Fig. 11. Breakdown of observer responses for 2D and DBT simulated images 

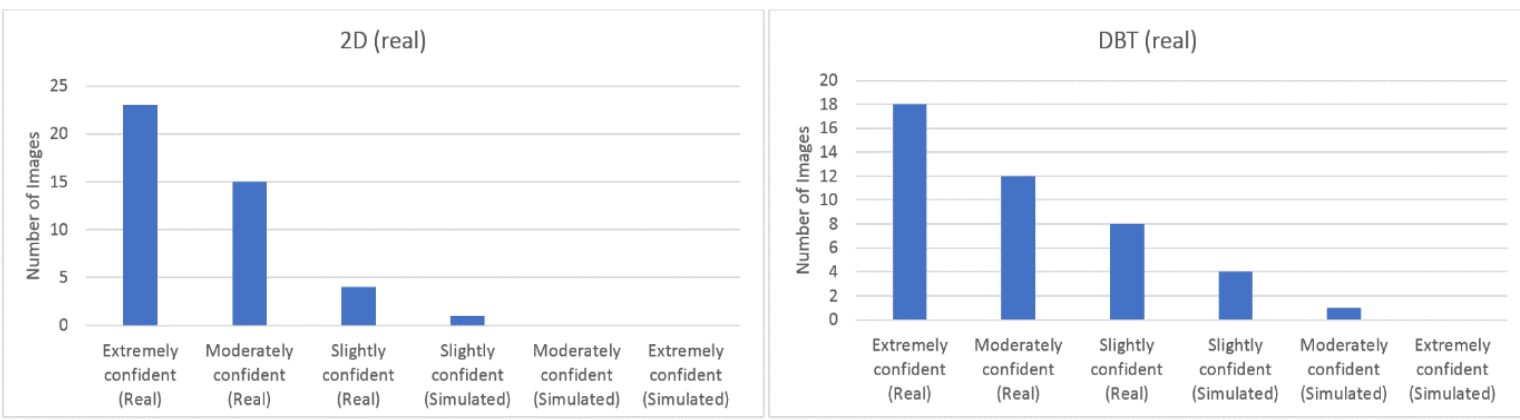

Fig. 12. Breakdown of observer responses for 2D and DBT real images

Table 1. Comparison of results between current study (with background blending) and previous study (without background blending) for lesions rated as real

\begin{tabular}{|c|c|c|}
\hline & 2D ratings & DBT ratings \\
\hline Spiculated lesions with background blending (this work) & $88.4 \%$ & $67.4 \%$ \\
\hline Spiculated lesions without background blending [10] & $60 \%$ & $50 \%$ \\
\hline
\end{tabular}

\section{CONCLUSION AND FUTURE WORK}

A method has been described for creating spiculated lesions and inserting these into 2D or DBT image volumes by distorting the surrounding local architecture. This has produced a realistic set of lesions suitable for use in AFC studies. The latest observer study results demonstrated that $88.4 \%$ of simulated images of lesions in 2D and $67.4 \%$ of simulated lesions in DBT were rated as definitely or probably real on a six-point scale. This presents a significant improvement on our previous work which did not employ any background blending algorithms to simulate spiculated lesions in clinical images. These results also show that a large proportion of simulated lesions look equally good in both 2D and DBT which is particularly challenging to achieve. Future work will involve adding more breast readers to the study and generating ROC curves.

\section{ACKNOWLEDGMENTS}

This work is part of the OPTIMAM2 project funded by Cancer Research UK (grant number: C30682/A17321). We are grateful to Hologic for the provision of the image processing and reconstruction software. We thank Professor Fiona Gilbert at Addenbrooke's Hospital Cambridge for providing access to images in the TOMMY trial database. 


\section{REFERENCES}

[1] Maidment, A "Virtual clinical trials for the assessment of novel breast screening modalities," IWDM 2014 Lecture Notes in Computer Science Publishing 8539, 1-8 (2014).

[2] Elangovan, P., Warren, L. M., Mackenzie, A., Rashidnasab, A., Diaz, O., Dance, D. R., Young, K. C., Bosmans, H., Strudley, C. J. and Wells, K., "Development and validation of a modelling framework for simulating 2D-mammography and breast tomosynthesis," Phys. Med. Biol. 59, 4275-4293 ( 2014).

[3] Rashidnasab, A., Elangovan, P., Diaz, O., Mackenzie, A., Young, K. C., Dance, D. R. and Wells, K., "Simulation of 3D DLA masses in digital breast tomosynthesis," Proc. SPIE 8668 Medical Imaging Physics of Medical Imaging, 86680Y (2013).

[4] Rashidnasab, A., Elangovan, P., Yip, M., Diaz, O., Dance, D. R., Young, K. C. and Wells, K., "Simulation and assessment of realistic breast lesions using fractal growth models," Phys. Med. Biol. 58, 5613-5627 ( 2013 ).

[5] Shaheen, E., Van Ongeval, C., Zanca, F., Cockmartin, L., Marshall, N., Jacobs, J., Young, K. C., Dance, D. R. and Bosmans, H., "The simulation of 3D microcalcification clusters in 2D digital mammography and breast tomosynthesis," Med. Phys. 38, 6659-6671 (2011).

[6] Elangovan, P., Mackenzie, A., Dance, D. R., Young, K. C., Cooke, V., Wilkinson, L., Given-Wilson, R. M., Wallis, M. G. and Wells, K., "Design and validation of realistic breast models for use in multiple alternative forced choice virtual clinical trials," Phys. Med. Biol. 62, 2778-2794 (2017).

[7] Mackenzie, A., Marshall, N. W., Hadjipanteli, A., Dance, D. R., Bosmans, H and Young, K. C., "Characterisation of noise and sharpness of images from four digital breast tomosynthesis systems for simulation of images for virtual clinical trials," Phys. Med. Biol. 62, 2376-2397 (2017).

[8] de Sisternes, L., Brankov, J. G., Zysk, A. M., Schmidt, R. A., Nishikawa, R. M., and Wernick, M. N. “A computational model to generate simulated three-dimensional breast masses," Med. Phys 42, 1098-1118 (2015).

[9] Berks, M., Taylor, C., Rahim, R., Boggis, C. and Astley, S., "Modelling structural deformations in mammographic tissue using the Dual-Tree complex wavelet,” Digital Mammography Lecture Notes in Computer Science 6136, 145-152 (2010).

[10] Elangovan, P., Alrehily, F., Ferrari Pinto, R., Rashidnasab, A., Dance, D. R., Young, K. C., and Wells, K., "Simulation of spiculated breast lesions ," Proc. SPIE 9783 Medical Imaging 2016: Physics of Medical Imaging, 97832E (2016).

[11] Halling-Brown, M., Looney, P., Patel, M. N., Warren, L. M., Mackenzie, A. and Young, K. C, "The oncology medical image database (OMI-DB)," Proc SPIE 9039 (2014). 